\title{
Understanding the PICUP community of practice
}

\author{
Paul W. Irving ${ }^{1}$ and Marcos D. Caballero ${ }^{1,2,3}$ \\ ${ }^{1}$ Department of Physics and Astronomy, Michigan State University \\ ${ }^{2}$ CREATE for STEM Institute, Michigan State University \\ ${ }^{3}$ Department of Physics and Center for Computing in Science Education, University of Oslo
}

\begin{abstract}
The mission of the Partnership for Integration of Computation into Undergraduate Physics (PICUP) is to expand the role of computation in the undergraduate physics curriculum. A primary tool to facilitate this expansion is the PICUP Faculty Development Workshop (FDW), which functions similarly to the New Faculty Workshop (NFW), in that invitees engage and learn about educational practices associated with integrating, in this case, computation into their curriculum designs. With this focus on community building and engagement in the legitimate peripheral practices of the PICUP community, we have developed a project that applies the communities of practice framework to understand how the PICUP community develops over time. Presented in this paper are the initial stages of this work that focuses on understanding the central member's perceptions of the communities goals and ways to participate in the community.
\end{abstract}

\section{INTRODUCTION}

The Partnership for Integration of Computation into Undergraduate Physics (PICUP) was formed as a response to a call to articulate and to discuss pedagogy, methodology, and issues relevant to integrating computation into undergraduate physics curricula. One of the guiding principles of the PICUP group is that a comprehensive integration of computation into the undergraduate physics curricula will require a supportive social network linking developers and users, including pioneers, early adopters, willing followers, and holdouts [1]. One of the initial efforts to follow this guideline and to begin a movement towards more comprehensive integration of computation into undergraduate physics is the development and running of the PICUP Faculty Development Workshop (FDW).

The PICUP FDW bears similarities to the New Faculty Workshop (NFW) [2] in its purpose of introducing faculty to curriculum and to pedagogical ideas in the hope that the attendees will then successfully implement those ideas when they return to their home institutions. A divergence between the two workshops goals is that the NFW leans towards promoting a research-based instructional strategy (RBIS) or multiple RBISs whereas whereas the PICUP FDW is more focused on attendee self-development of computational activities. This difference in focus can be attributed to the fact that although the workshops may have similar purposes, the curriculum and pedagogical ideas are focused on achieving different goals. The NFW aims to promote the usage of research-based teaching norms (which, could include computationally based curriculum), and the PICUP FDW focuses on providing faculty the support to develop their own activities.

We focus on the parallel between the PICUP FDW and NFW because of the NFWs' centrality to a comprehensive research program examining faculty use of RBIS by Dancy and Henderson [3, 4]. Their research built a broad outline of the challenges that faculty face when trying to implement RBISs at their institutions while also examining when during the implementation phase faculty discontinue use of an
RBIS. They highlight that although the model of dissemination of RBISs is successful at the level that faculty are aware of their existence, it tends not to be supportive when a physics faculty member is making decisions about implementation at their institution and when they encounter barriers during that implementation. This lack of support is particularly salient for faculty trying to overcome institutional barriers when attempting the implementation of a RBIS. One of the ways that the NFW organizers are working to combat this lack of support at crucial times during the application process is the development of support networks. These support systems often manifest in the form of online communities, which offer faculty the opportunity to have their problems acknowledged and to troubleshoot by faculty members in similar situations - ideally in similar contexts.

The PICUP FDW, influenced by Dancy and Henderson's work, supports a similar approach to community development through the use of a cloud-based team collaboration tool and service called SLACK and online support group checkins. This community development pushes towards a dualpronged goal of both supporting integrators while also growing the PICUP community of practice. The fact that the NFW and the PICUP FDW focus on developing support communities invited an alternative approach to examining curriculum change and the development of a community of people pushing for the common goal of integration of computation into the undergraduate curriculum. By examining the PICUP FDW and its attendee's roles and practices from the perspective of the communities of practice (COP) framework, we aim to understand how communities of support develop from the PICUP FDW, but also how the PICUP community grows in both numbers and its actions over the four-year duration of this research project.

\section{COMMUNITIES OF PRACTICE FRAMEWORK}

COP's [5] have three main features: (1) the individuals within form a group, either co-located or distributed, (2) the group has common goals or shared enterprise, and (3) the 
group shares and develops knowledge focused on common practices [6]. From this perspective a COP can be considered to be made up of 3 separate parts: the domain, the community and the practice. In this study the focus will be on investigating the domain and the practice of the PICUP COP. The domain is essentially what shared competencies do the members of the community have that distinguishes them from people not members of the community. Practice can be distinguished by the ways that community members engage in the legitimate peripheral practices (LPP) of that community. The practices that are recognized as being a legitimate part of the community. Communities are split into different levels of participation and members of the community are either central participants or peripheral participants. Peripheral members can be found to be at different points in their trajectory towards central membership (although that does not imply they will reach central membership or that they could stop participating in the community at some point), and they become more central by engaging in the LPP's of the community while being guided by central participants. These LPP's can be considered transactional, peripheral, occasional, active or core and we will use these classifications later when discussing the practices that members were found to engage it in the PICUP community.

In this paper, we are arguing that the PICUP FDW organizers are representative of central participants in the PICUP $\mathrm{COP}$ and attendees are guided into more central (the level of centrality may be different for each attendee) membership by attending the workshop. Situationally, this work is positioned before the first PICUP FDW and is part of a larger ongoing study into PICUP community development. The focus of this paper is trying to understand the domain and roles of the PICUP COP as defined by the central participants. Through the central participants' beliefs and perceptions, we can assess whether the community does share a common goal (essential for the application of the COP framework). We also examine what the central community members view as the LPP's and roles of participants in the PICUP COP.

\section{DATA AND ANALYSIS}

Semi-structured interviews were conducted with the five organizers of the PICUP FDW starting from the supposition that these five members represent key central members of the PICUP community of practice. Because there are only five interviewees and the organizers are easily Google-able, we have given each interviewee a gender neutral name and will only provide personal information if entirely pertinent to the presentation of the data. This approach would not be our typical approach to presenting interview data as we feel that both gender and background are typically essential to include in the presentation of any interview data. But the circumstances require this approach to data presentation.

The interviewees were interviewed two weeks before the first PICUP FDW with the discussion focusing on their back- ground, experiences with computation and computational curriculum design, their goals for the community, and their thoughts on the importance of community development. The interviewees span a variety of backgrounds and career positions. Each interview was transcribed and then analyzed in a thematic analysis [7] approach with a focus on both preconceived and emergent themes. Due to the importance of the central members of a community having a shared goal, it was important to directly investigate the central members goals for the PICUP COP. Their goals became the focus of a line of questioning in each interview to ensure that it was appropriate to apply the COP framework to the PICUP community. Interviewees' responses to this line of questioning were analyzed for shared themes and alternative perspectives and are presented in Sec. III A. The other theme that emerged from the data, which is displayed in Sec. III B, is the various perceived roles and related ways of participating in the community. These roles emerged from analysis of interviewees' perceptions of their role, discussions around how the community will grow, and the practices that attendees will engage in at the PICUP FDW. Common themes were identified and then categorized and are presented later in Sec. III B.

\section{A. Common goals of the PICUP community}

A theme that was intentionally explored as part of this initial interview set was whether these five central members shared common goals for the PICUP community of practice. From a theoretical perspective, different goals could call into question the use of the COP framework. From a practical standpoint, different goals could cause mixed messaging at the PICUP FDW, which could have effects on how the community grows.

Unsurprisingly, the five central members did have a shared goal for the PICUP community - the student-centered goal of having students experience computation in their undergraduate curriculum.

Sam: We want students to understand the intersection of computing and physics.

But there was a degree of nuance to this central goal found in the differing motivations for having students gain an experience of computation in physics. For some of the central members, students gaining computational experience is motivated by the fact that the majority of physics majors do not end up in a physics position after graduation. The argument they make is that these students are currently under-prepared for their future career that will more than likely involve some form of computation.

Corey: Because of what most BS physics majors do when they get out, period. And it's not going on to replicate ourselves. They work in labs...I want them to realize computation is their connection to all the sciences, not just their own, because most of them will not be...working in their own.

The emphasis on the assertion that the goal is not to repli- 
cate another physicist is interesting. Particularly, when it is taken in parallel with the claim by multiple of the interviewees that part of the goal of integrating computation is to add a level of authenticity for students for what it means to do physics.

Sam: I guess I no longer think of it as computation and physics, I think of it as doing physics. Like to do physics and to understand physics you have to understand computing and physics at this point.

The two perspectives, that of computational integration in physics as preparation for a non-physics career and that of computational integration to provide an authentic experience of doing physics are not incompatible together. Potentially though, over time, if enough people feel centered within one of these perspectives, this could result in a division in the PICUP community of practice to a community that is focused on developing computational skills in introductory physics classrooms and a community that is focused on exploring authentic physicist experiences in upper division physics classes. Other motivations described for including computation in physics include the affordances that computation provides for the development of conceptual understanding via visualizations and that it emphasizes the important practice of modeling.

Of these motivations, the emphasis on modeling was the only one mentioned by more than one of the central members.

\section{B. Participants in the PICUP Community of Practice}

The second theme which emerged from the analysis of the data is the perceived different roles that both newcomers, current members, and central members occupy, or can fill in the PICUP COP. The following roles could act as stepping stones towards central membership of the community except it is unclear at this moment whether an advocate is more central to the community than a researcher or vice versa. The roles are Interested Party, User, Contributor, Guide, Advocate and Researcher.

Interested Party - Several of the central members discuss the fact that the message is "out" about computation already and that there are plenty of faculty members who are interested in integrating computation, but those faculty do not yet have the tools to do so.

Jamie: I was going to say convincing people that it's important. I have a sense that most people know that it's important. They're just - they don't know how to integrate it...so what we're trying to do is make it easier for people.

These faculty members are still members of the community but they are on the outer rim of membership and are currently labeled as Interested Parties which aligns with the transactional level of participation from Wengers work.

User - The next level up of membership is the users, these are people who are perceived to be using the materials being developed by the PICUP COP. The PICUP website has a repository of computational activities that are accessible to everyone for a variety of class levels and audiences. Users are essentially faculty who are using these materials or others without contributing their own to the community. This role aligns with the occasional participants from Wengers research.

Contributor - A big emphasis of the PICUP FDW is to encourage participants to become contributors who upload their own activities to the PICUP website. This focus on development manifests in the PICUP FDW schedule with the whole week being focused around each attendee producing a product that they will then implement in their classroom in the Fall.

Riley: And by having them drink the Kool Aid, so to speak, they are automatically becoming part of that community...hopefully they will not just do these things in their classes but also contribute

One of the main focuses of the PICUP FDW is that attendees leave the workshop having reached the point of contributor in the PICUP COP. This role aligns with the occasional participants from Wengers research.

Guide - As with the adoption of RBISs, there are often a lot of potential barriers to success when trying to integrate computation into your curriculum for the first time. It is the guides responsibility to help members overcome these barriers. Many of the barriers discussed by Henderson and Dancy are shared, such as situational barriers like content coverage and class size, but there are also shared barriers that become complicated with computation:

Jamie: It's certainly been a learning experience...just trying to figure out... what the right level is. You know, still trying to push the students...but not overwhelm them.

There is an added layer of difficulty to curriculum design when it comes to computation as the difficulties can lie not just in the physics, but in the students having the necessary computational skills to be able to engage with the physics through computation. The central members indicate that members of the community need to take on the role of guides.

Jamie: And so I think hearing from all of these people is very important...I think the different ways of entering computation, then the more likely it is to have something resonate with someone, and say, "Well, hey, I want to do it that way." And, you know, that leads to questions and that leads to someone seeing that this is doable in terms of the time commitment.

The guide role is also being interwoven into the ongoing supports, such as the use of SLACK and the video chat meet ups that are planned for after the FDW.This role aligns with the active participants from Wengers research.

Advocate - There has been great deal of effort put into creating a community around the integration of computation into the undergraduate curriculum both before and after the formation of PICUP. However, this community is still relatively small in comparison to, for example, the PER community. 
The central members that were interviewed view members of the community becoming advocates as an important step in the growth of the community.

Dylan: I mean, a valid entity, and it has to be vibrant and successful [the PICUP community]. It has to demonstrate that there's benefits to being a part of it. But, then, also, it has to have...That will only happen through...I don't know. What would you call it? This friendship evangelism mode that I've described or grassroots way of convincing. Start with the friendly colleagues, those who are open.

Dylan is articulating what several of the central members described - advocacy from a bottom-up perspective where attendees who have attended the PICUP FDW go home to their institutions and advocate for integration by displaying the benefits for colleagues who are perceived to be friendly.

Researcher - A parallel to the work of the NFW and the PER community that does not exist in the PICUP community is a large body of research that provides compelling evidence of the effectiveness of using computation to learn physics.

Sam: We don't actually understand what students understand about this stuff, we don't actually understand how students use that knowledge in different ways, and we don't understand... what are the appropriate ways of actually trying to help students understand it...does it make sense to have clicker questions? Does it make sense to have tutorials around this?...It's a fundamentally different thing, it's a paradigm shift for how you think about physics.

It is an open question as to whether Sam's questions need to be answered in order for the PICUP community to prosper depending on what potential adopters need to become interested parties. It is unclear how the advocate and researcher roles translates to Wenger's work. Advocates would seem to be core members whereas researcher still fits into a grey area because they could fall into multiple of Wengers levels of participation. It is important to note that Sam is talking about research from the perspective of understanding for implementation - so it is material that could be used to advocate for computational inclusion.

\section{DISCUSSION AND CONCLUSION}

Examining the PICUP FDW and COP from the perspective of the COP framework could allow us to answer multiple research questions. What is the role of workshops in community development? What aspects of a workshop's design propagate through to the attendees and how does this influence their practice going forward? How does a community based around curriculum reform develop over time? The research presented in this paper is an attempt to begin to answer these questions by starting with exploring the PICUP community from the perspective of central members just before the first implementation of the PICUP FDW. From the domain perspective, we have identified that, in general, the central members share the same common goal but with sometimes differing motivations. The fact that they share the common goal is an unexciting but necessary finding for the application of the communities of practice framework in this context.

We have also identified six different roles that community members can engage in from the perspective of the central participants. These roles need to be defined further through interviews with more peripheral members of the community. But they do seem to relate to the levels of participation indicated in Wenger's research although the correlation between the two for all roles is not entirely clear. For example, advocacy needs to be investigated further as the central members seem to switch between advocacy for actually integrating computation into one's instruction and advocacy for becoming more central members of the PICUP community. The researcher role is also worth further investigation given the often contentious relationship that researchers and potential adopters have in the PER community. Potential adopters often feel as though researchers are criticizing their teaching and are offering unrealistic fit-all-contexts solutions. Whereas the PICUP community perceive that they are only seeking integration for something that they believe non-PER friendly faculty already value. This belief brings into question the role of the researcher in this community. Are studies indicating the role of computation in the development of conceptual understanding needed? Is it sufficient to merely use the argument that potential adopters already use computation in their work as a physicist so why shouldn't be included?

Finally, filling out the space of roles within the PICUP community provides a ground floor for understanding the positions new members to the community will occupy while also allowing for a tracking of individual development within the community over time and how they identify as members of the community.
[1] N. Chonacky and D. Winch, American Journal of Physics vol. 76, 2008, pp. 327-333.

[2] https://www.aapt.org/Conferences/newfaculty/nfw.cfm. Retrieved 07/05/2017.

[3] M. Dancy and C. Henderson, American Journal of Physics vol. 78, 2010, pp. 1056-1063.

[4] C. Henderson and M. Dancy, Phys. Rev. Phys. Rev. ST Physics Ed. Research 8, 020104 (2012).
[5] Lave, J., and Wenger, E. (1991). Situated learning: Legitimate peripheral participation. New York: Cambridge University Press.

[6] P. Irving and E. Sayre, Phys. Rev. Phys. Rev. ST Physics Ed. Research 10, 010109 (2014).

[7] R. E. Boyatzis, Transforming qualitative information: Thematic analysis and code development. Thousand Oaks: Sage Publications. 